Check for updates

Cite this: RSC Adv., 2022, 12, 6459

\title{
Structure and absolute configuration of liquid molecules based on adamantane derivative cocrystallization $\dagger$
}

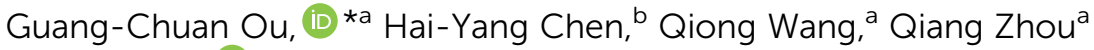 \\ and Fei Zeng (D) *a
}

Liquid molecules are difficult to crystallize, and their structures and absolute configurations cannot be directly determined by X-ray crystallography. We herein report the rapid cocrystallization of tetraaryladamantanes with liquid molecules. The structure of the liquid small molecules can be obtained by determining the crystal structure of the cocrystallized compound. The absolute configuration of chiral molecules can also be assigned, which cannot be accomplished by other methods such as nuclear magnetic resonance. In this paper, liquid compounds such as phenylethanol and phenylpropanol derivatives were selected. 1,3,5,7-Tetrakis(2,4-diethoxyphenyl)adamantane (TEO) powder was heated and dissolved in liquid molecules and allowed to stand overnight to undergo cocrystallization. The results show that the single-crystal structures and the absolute configurations of 16 liquid molecules were determined by cocrystallization, and the homochiral natures of chiral compounds were confirmed by solid circular dichroism spectral measurements.

Received 23rd December 2021
Accepted 31st January 2022

DOI: $10.1039 / \mathrm{d} 1 \mathrm{ra0} 2828 \mathrm{~g}$

rsc.li/rsc-advances sponges reported thus far are based on metal-organic frameworks, and their limitations are as follows: first, when the size of the target molecule is larger than one of the pores, the target molecule does not enter the crystal sponge. Second, it is difficult for hydrophilic molecules to enter the hydrophobic pores, and the "crystal sponge method" has been mainly applied to the determination of hydrophobic molecular structures. Several methods are being pursued to address these limitations. Recently, another particularly significant method using "crystallization chaperones" was presented. These crystallization chaperones, including tetraaryladamantanes ${ }^{8-19}$ and trimesic acid, ${ }^{20-27}$ readily form crystalline inclusion complexes with a range of guest molecules and provide accurate structural information on difficult-to-crystallize small molecules. Meanwhile, the high-throughput nanoscale crystallization of organicsoluble small molecules (encapsulated nanodroplet crystallization) was reported. ${ }^{28}$ However, the cocrystallization of universal chaperones with various small molecules, such as porphyrins, coworkers. $^{1-7}$ The crystal sponge most frequently employed in their studies was the metal-organic framework $\left\{\left[\left(\mathrm{ZnI}_{2}\right)_{3}(\operatorname{tris}(4-\right.\right.$ pyridyl)-1,3,5-triazene $\left.)_{2}\right]_{x}$ (solvent) $\}_{n}$. However, the crystal

${ }^{a}$ College of Chemistry and Bioengineering/Key Laboratory of Comprehensive Utilization of Advantage Plants Resources in Hunan South, Hunan University of Science and Engineering, Yongzhou, Hunan 425199, P.R. China. E-mail: ogcouguangchuan@163.com; zengfei@iccas.ac.cn

${ }^{b}$ Medical College, Guangxi University, Nanning, Guangxi 530004, P.R. China. E-mail: czyzsu@gxu.edu.cn

$\dagger$ Electronic supplementary information (ESI) available. CCDC 2126622 to 2126637 for 1-16. For ESI and crystallographic data in CIF or other electronic format see DOI: $10.1039 / \mathrm{d} 1 \mathrm{ra} 09284 \mathrm{~g}$

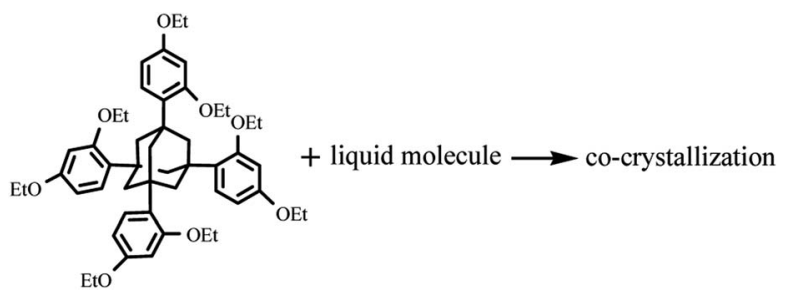

Scheme 1 Cocrystallization of TEO with liquid small molecules. 
calixarenes, or cyclodextrins, with a proven ability to obtain the structure of a broad range of different organic molecules is yet to be reported. ${ }^{12}$ Here, we report the rapid cocrystallization of 1,3,5,7-tetrakis(2,4-diethoxyphenyl)adamantane (TEO) with liquid small molecules (Scheme 1), such as phenylethanol and phenylpropanol derivatives, and determine the absolute configuration of chiral small molecules.

\section{Experimental section}

\section{Materials and physical measurements}

1,3,5,7-Tetrakis(2,4-diethoxyphenyl)adamantane (TEO) was customized by WuXi AppTec (Tianjin, China). All of the other chemicals are commercially available and used without further purification. The solid circular dichroism (CD) spectra were recorded in the 200-350 $\mathrm{nm}$ region using a JASCO J-815 spectropolarimeter.

\section{General methods for cocrystallization}

Approximately $2 \mathrm{mg}$ of solid TEO $(0.0025 \mathrm{mmol})$ in a cylindrical sample bottle $(1 \mathrm{~mL})$ was added to approximately $40 \mathrm{mg}$ of liquid analyte ( 2 drops). The resulting suspension was heated on a hot plate until a clear solution formed. The resulting solution was allowed to cool to room temperature with the sample bottle on the plate. After $12 \mathrm{~h}$, colorless prism- or blockshaped crystals were obtained and then analysed by X-ray single-crystal diffraction.

\section{X-ray crystallography}

Single-crystal data were collected on a Bruker Apex II diffractometer at $296 \mathrm{~K}$ using $\mathrm{Mo}-\mathrm{K} \alpha$ radiation $(\lambda=0.71073 \AA)$ or on a SuperNova Dual AtlasS2 diffractometer at $150 \mathrm{~K}$ using $\mathrm{Cu}-\mathrm{K} \alpha$ radiation $(\lambda=1.54184 \AA)$. All empirical absorption corrections were applied by the SADABS program ${ }^{29}$ or CrysAlisPro (Rigaku, V1.171.39.46, 2018). The structures were solved by direct methods, which yielded the positions of all nonhydrogen atoms. These were refined first isotropically and then anisotropically. All nonhydrogen atoms were refined anisotropically. Hydrogen atom positions were calculated geometrically and refined using the riding model. All calculations were performed using the SHELXTL system of computer programs. ${ }^{30}$ Most of the guest molecules are disordered in cocrystallization. The crystallographic data are summarized in Table S1. $\uparrow$ CCDC reference numbers 2126622 to 2126637 for 1-16, respectively. See http://www.rsc.org/suppdata/ for crystallographic data in CIF or other electronic format.

\section{Results and discussion}

We chose structurally similar liquid analytes, such as phenylethanol and phenylpropanol derivatives, for cocrystallization experiments with TEO. A mixture of the analytes and TEO without any solvent was heated for approximately $30 \mathrm{~s}$ on a laboratory hotplate until a clear solution formed. The resulting solution was left to cool to room temperature. Colourless crystals for X-ray diffraction were obtained after crystallization overnight, and the crystal structures of the liquid analytes were confirmed by X-ray crystallography. With most guest molecules, triclinic crystal systems and a host/guest ratio of $1: 1$ were found. However, with 1-(4-chlorophenyl)ethanol and 2-(4methylphenyl)-1-propanol, TEO crystallized in a trigonal crystal system (Table $\mathrm{S} 1 \dagger$ ).

Successful examples can be found from the full list of 16 liquid small molecules tested in this study (Table 1), including a series of phenylethanol derivatives (1a-1e) (Fig. 1), phenylpropanol derivatives (2a-2d) (Fig. 2), ethylbenzylamine (3a-3c)

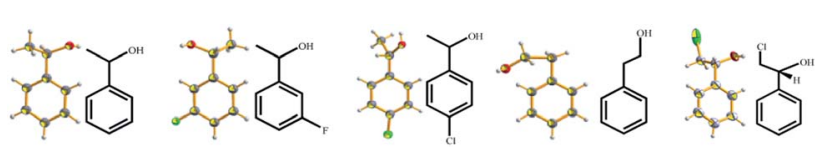

Fig. 1 X-ray crystal structures of liquid phenylethanol derivatives obtained by cocrystallization with TEO (ORTEP plots of the encapsulated molecules at the $50 \%$ probability level).

Table 1 Cocrystallization experiments of TEO and analytes

\begin{tabular}{|c|c|c|c|c|c|}
\hline Serial No. & Analytes & Crystal system & $R 1$ & Flack values & Sample No. \\
\hline 1 & Phenylethanol & Triclinic & 0.064 & & 1a \\
\hline 2 & 1-(3-fluorophenyl)ethanol & Triclinic & 0.060 & & $1 b$ \\
\hline 3 & 1-(4-chlorophenyl)ethanol & Trigonal & 0.081 & & $1 \mathrm{c}$ \\
\hline 4 & 2-Phenylethanol & Triclinic & 0.074 & & 1d \\
\hline 5 & $(R)-(-)$-2-chloro-1-phenylethanol & Triclinic & 0.096 & $0.124(14)$ & 1e \\
\hline 6 & 1-Phenyl-1-propanol & Triclinic & 0.066 & & $2 \mathbf{a}$ \\
\hline 7 & $(S)-(-)-1$-phenyl-1-propanol & Triclinic & 0.045 & $-0.01(4)$ & $2 \mathbf{b}$ \\
\hline 8 & $(R)-(+)-1$-phenyl-1-propanol & Triclinic & 0.041 & $0.04(3)$ & $2 c$ \\
\hline 9 & 2-(4-methylphenyl)-1-propanol & Trigonal & 0.071 & & $2 d$ \\
\hline 10 & $\alpha$-ethylbenzylamine & Triclinic & 0.076 & & $3 \mathbf{a}$ \\
\hline 11 & $(R)-(+)$ - $\alpha$-ethylbenzylamine & Triclinic & 0.048 & $0.06(5)$ & $3 \mathbf{b}$ \\
\hline 12 & $(S)-(-)$ - $\alpha$-ethylbenzylamine & Triclinic & 0.067 & $0.03(8)$ & $3 c$ \\
\hline 13 & 2-Chloropyridine & Triclinic & 0.062 & & $4 a$ \\
\hline 14 & 3-Chloropyridine & Triclinic & 0.066 & & $4 \mathbf{b}$ \\
\hline 15 & Styralyl acetate & Triclinic & 0.065 & & 5 \\
\hline 16 & Methacrylic acid & Triclinic & 0.097 & & 6 \\
\hline
\end{tabular}



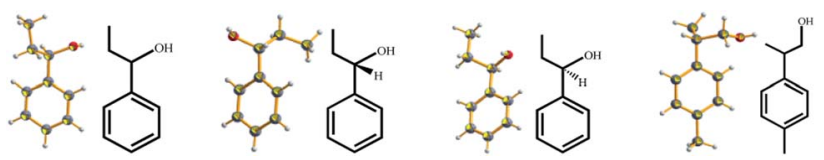

Fig. 2 X-ray crystal structures of liquid phenylpropanol derivatives obtained by cocrystallization with TEO (ORTEP plots of the encapsulated molecules at the $50 \%$ probability level).
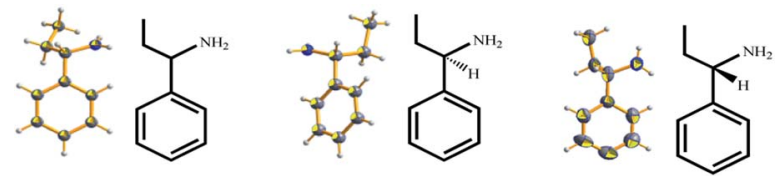

Fig. 3 X-ray crystal structures of liquid $\alpha$-ethylbenzylamine obtained by cocrystallization with TEO (ORTEP plots of the encapsulated molecules at the $50 \%$ probability level).

(Fig. 3), isomers such as 2-chloropyridine (4a) and 3-chloropyridine (4b), styralyl acetate (5) used in fragrance and flavor, and methacrylic acid (6) (Fig. 4) applied to organic chemical raw materials and intermediates of polymers (Fig. 5). There are no covalent bonds or strong mutual interactions, such as hydrogen bonding between host and guest molecules in the asymmetric unit of the complex (Fig. 6). The liquid small molecules solidified and crystallized with the aid of TEO, and most of the liquid molecules were disordered due to the lack of mutual interactions with the host during cocrystallization. TEO can rapidly crystallize, perhaps due to its shape and symmetry, in which alkoxy groups can adopt different conformations to accommodate different guest molecules. ${ }^{14}$ However, a crystalline sponge needs to be preformed with a suitable crystal lattice for accommodating analytes. TEO solvated by liquid molecules can crystallize without any special interactions and encapsulate
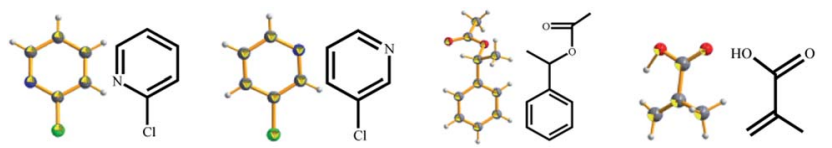

Fig. 4 X-ray crystal structures of other liquid analytes obtained by cocrystallization with TEO (ORTEP plots of the encapsulated molecules at the $50 \%$ probability level).
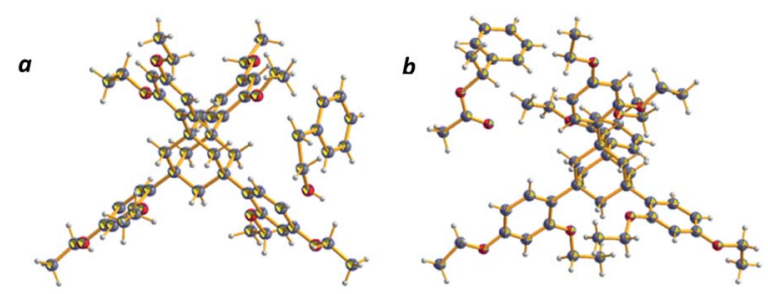

Fig. 5 Structure of representative cocrystals and 50\% probability displacement ellipsoids for non-H atoms: (a) 2-phenylethanol/TEO and (b) styralyl acetate/TEO.

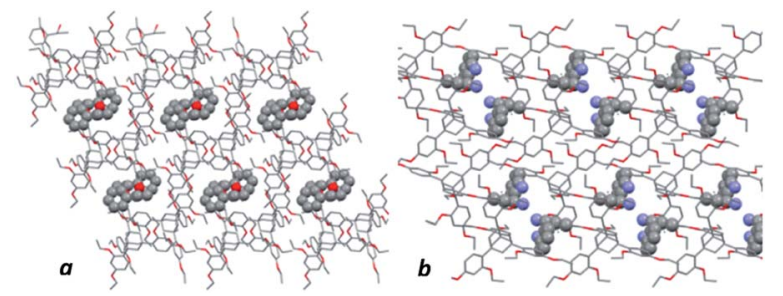

Fig. 6 Packing arrangement in representative cocrystals: (a) 1-phenyl1-propanol/TEO and (b) $\alpha$-ethylbenzylamine/TEO.

a broad range of molecules and would be a potential candidate for extended crystalline sponges.

Structurally similar liquid phenyl alcohols or amines were selected, including two pairs of enantiomers, namely, $(R)$ - and $(S)$-1-phenyl-1-propanol (2b and $2 \mathbf{c}$ ) and $(R)$ - and $(S)$ - $\alpha$-ethylbenzylamine ( $\mathbf{3 b}$ and $\mathbf{3 c}$ ). Flack parameters were in the range of $-0.01(4)$ to $0.124(14)$ (Table 1), allowing for the unambiguous assignment of absolute configuration. The absolute structure parameters are critical for determining the correct absolute configuration of the chiral guest molecule. The initial structural model was solved using SHELXTL programs, and the intensity data appeared to be centrosymmetric due to the strong reflexes originating from host molecules, although the encapsulated guest molecule was chiral. To resolve this conflict, it is necessary to introduce particular treatments according to previous reports. ${ }^{18}$ Compared to the above reports, the homochiral natures of chiral compounds were further confirmed by solid CD spectrum.

\section{Circular dichroism spectra}

The crystal samples were filtered, dried, and determined after grinding and pressing on a JASCO J-815 spectropolarimeter. The results of the selected solid circular dichroism measurements are consistent with the results of the X-ray crystal structures. As shown in Fig. 7a, the bulk crystals of $\mathbf{3 b}$ showed negative and positive Cotton effects at 207 and $273 \mathrm{~nm}$, respectively, and those of $3 \mathbf{c}$ also showed positive (208 and $231 \mathrm{~nm}$ ) and negative Cotton effects $(296 \mathrm{~nm})$. The selected corresponding UV spectra of $\mathbf{3 b}$ in Fig. S1. $\dagger$

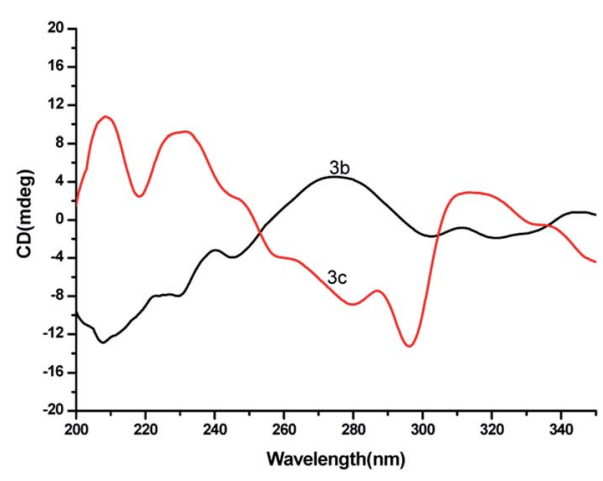

Fig. 7 Solid CD spectra of TEO-S- $\alpha$-ethylbenzylamine (3b) and TEO$R$ - $\alpha$-ethylbenzylamine (3c). 


\section{Conclusions}

In summary, we report a rapid method for the structure determination of liquid small molecules. These liquid small molecules can cocrystallize rapidly with 1,3,5,7-tetrakis(2,4diethoxyphenyl)adamantane without covalent bonds or strong directional interactions and can be used to determine the absolute configuration of chiral liquid molecules. Unfortunately, most liquid molecules are disordered during cocrystallization, increasing the difficulty of structural analysis. 1,3,5,7Tetrakis(2,4-diethoxyphenyl)adamantane can cocrystallize rapidly with liquid molecules and encapsulate a broad range of molecules, demonstrating its potential application in extended crystalline sponges.

\section{Conflicts of interest}

There are no conflicts to declare.

\section{Acknowledgements}

This work is supported by the National Natural Science Foundation of China (51772091), the Scientific Research Fund of Hunan Provincial Education Department (20A210), and the Construct Program of Applied Characteristic Discipline in Hunan University of Science and Engineering.

\section{Notes and references}

1 Y. Inokuma, S. Yoshioka, J. Ariyoshi, T. Arai, Y. Hitora, K. Takada, S. Matsunaga, K. Rissanen and M. Fujita, Nature, 2013, 495, 461.

2 T. Kawamichi, T. Haneda, M. Kawano and M. Fujita, Nature, 2009, 461, 633.

3 Y. Inokuma, S. Yoshioka, J. Ariyoshi, T. Arai and M. Fujita, Nat. Protoc., 2014, 9, 246.

4 S. Yoshioka, Y. Inokuma, M. Hoshino, T. Sato and M. Fujita, Chem. Sci., 2015, 6, 3765.

5 N. Zigon, M. Hoshino, S. Yoshioka, Y. Inokuma and M. Fujita, Angew. Chem., Int. Ed., 2015, 54, 9033.

6 Y. Inokuma, M. Kawano and M. Fujita, Nat. Chem., 2011, 3, 349.

7 G. A. O'Brien, A. Maruyama, Y. Inokuma, M. Fujita, P. S. Baran and D. G. Blackmond, Angew. Chem., Int. Ed., 2014, 53, 11868.

8 J. L. Johnson, K. C. Entzminger, J. Hyun, S. Kalyoncu, D. P. Heaner Jr, A. Morales, A. Sheppard, J. C. Gumbart, J. A. Maynard and R. L. Lieberman, Acta Crystallogr., Sect. D: Biol. Crystallogr., 2015, 71, 896.
9 M. B. Bhatt and G. R. Desiraju, CrystEngComm, 2008, 10, 1747.

10 M. P. Bryn, C. J. Curtis, Y. Hsiou, S. I. Khan, P. A. Sawin, S. K. Kendrick, A. Terzis and C. E. Strouse, J. Am. Chem. Soc., 1993, 115, 9480.

11 T. Gruber, C. Fischer, W. Seichter, P. Bombicz and E. Weber, CrystEngComm, 2011, 13, 1422.

12 M. Ceborska, Chem. Phys. Lett., 2016, 651, 192.

13 Y. Li, S. Tang, A. Yusov, J. Rose, A. N. Borrfors, C. T. Hu and M. D. Ward, Nat. Commun., 2019, 10, 4477.

14 A. Schwenger, W. Frey and C. Richert, Chem.-Eur. J., 2015, 21, 8781.

15 P. E. Alexandre, A. Schwenger, W. Frey and C. Richert, Chem.-Eur. J., 2017, 23, 9018.

16 A. Schwenger, W. Frey and C. Richert, Angew. Chem., Int. Ed., 2016, 55, 13706.

17 F. Krupp, S. He, W. Frey and C. Richert, Synlett, 2018, 29, 1707.

18 F. Krupp, W. Frey and C. Richert, Angew. Chem., Int. Ed., 2020, 59, 15875.

19 F. Rami, J. Nowak, F. Krupp, W. Frey and C. Richert, Beilstein J. Org. Chem., 2021, 17, 1476-1480.

20 S. V. Kolotuchin, E. E. Fenlon, R. W. Wilson, C. J. Loweth and S. C. Zimmerman, Angew. Chem., Int. Ed. Engl., 1995, 34, 2654.

21 S. V. Kolotuchin, E. E. Fenlon, R. W. Wilson, C. J. Loweth and S. C. Zimmerman, Angew. Chem., Int. Ed. Engl., 1995, 34, 2654.

22 Y. C. Yan, B. M. Kariuki, C. E. Hughes, A. J. Logsdail and K. D. M. Harris, Cryst. Growth Des., 2020, 20, 5736.

23 A. Ibenskas, M. Simenas and E. E. Tornau, J. Phys. Chem. C, 2016, 120, 6669.

24 M. R. Goldyn, D. Larowska and E. Bartoszak-Adamska, Cryst. Growth Des., 2021, 21, 396.

25 L. Rajput, N. Jana and K. Biradha, Cryst. Growth Des., 2010, 10, 4565.

26 S. Bhattacharya and B. K. Saha, Cryst. Growth Des., 2011, 11, 2194.

27 G. C. Ou, Q. Wang, Q. Zhou and X. F. Wang, Crystals, 2021, 11, 409.

28 A. R. Tyler, R. Ragbirsingh, C. J. McMonagle, P. G. Waddell, S. E. Heaps, J. W. Steed, P. Thaw, M. J. Hall and M. R. Probert, Chem, 2020, 6, 1755.

29 G. M. Sheldrick, SHELXL-2014/7: program for the solution of crystal structures, University of Göttingen, Göttingen, Germany, 2014.

30 G. M. Sheldrick, Acta Crystallogr., Sect. C: Struct. Chem., 2015, $71,3$. 